\title{
LA CREATIVIDAD COMO FACTOR PARA EL ÉXITO ACADÉMICO Y LA CALIDAD DE VIDA DE ESTUDIANTES UNIVERSITARIOS
}

\author{
CREATIVITY AS A FACTOR IN ACADEMIC SUCCESS \\ AND QUALITY OF LIFE FOR UNIVERSITY STUDENTS
}

Edith Alejandra Pérez Márquez*

\section{Resumen}

La creatividad puede utilizarse como método para lograr el éxito académico y promover la calidad de vida en los estudiantes universitarios. Este estudio, de tipo descriptivo, transeccional y no experimental tiene como objetivo analizar descriptivamente la creatividad, éxito académico y calidad de vida, para identificar la situación actual del estudiante universitario. Para ello se diseñó y aplicó el instrumento de investigación CEECaVi a 164 estudiantes de las Unidades Académicas de Ingeniería, Enfermería y Psicología una Universidad pública de México, analizando las variables con 107 ítems. Como principales resultados se obtiene que los estudiantes demostraron que se enfocan en la realización de proyectos de manera innovadora; son flexibles, curiosos y espontáneos; y conocen la importancia de mantenerse saludables tanto física como emocionalmente. Se concluye que el uso de la creatividad es trascendental, siendo su adversario la procrastinación, promovida por el exceso de trabajo y las emociones desagradables.

\section{Palabras clave:}

Creatividad, gestión de la educación, calidad de vida, estudiante universitario, proyecto del alumno, salud mental.

* Doctorado en Ciencias de la Educación, Universidad Autónoma de Zacatecas, Zacatecas, Zac. México. email: eapm@uaz.edu.mx, ORCID https://orcid.org/0000-0001-5724-4354 


\begin{abstract}
Creativity may be used as a method to achieve academic success and promote quality of life among tertiary students. This descriptive, transactional, and no experimental study aims at investigating creativity, academic achievement and quality of life to identify the current situation of university students. Thus, the research tool CEECaVi was designed and applied to 164 students from the faculty of Engineering, Nursing, and Psychology from a public university of Mexico, analyzing the research variables with 107 items. Results suggest students demonstrated that they focus on carrying out projects in an innovative way, they were flexible, curious and spontaneous, and they knew the importance of staying healthy both physically and emotionally. It is concluded that the use of creativity is transcendental and its adversary is procrastination promoted by overwork and unpleasant emotions.
\end{abstract}

\title{
Keywords:
}

Creativity, educational management, quality of life, university student, student's project, mental health.

\section{Introducción}

En la vida cotidiana de las personas, se encuentra presente la creatividad en diversas formas, se resuelven problemas y se realizan proyectos con un propósito, ya sea como medio de mejorar su condición, facilitar las tareas y/o la comodidad de la estética para armonizar el medio en el que vive. La creatividad se trata de un proceso psicológico superior del sistema nervioso central.

Se entiende la creatividad como una innovación valiosa y creación reciente; se define como la capacidad de dar origen a algún producto o contenido nuevo, desconocido y valioso, con sentido de ética, que tenga valores morales y busque el bien común entre las personas, así como también es la habilidad para encontrar, tanto nuevos y mejores modos de realizar actividades, como diversas combinaciones de la información, a lo que también se agrega la 
flexibilidad, originalidad y fluidez. Puede pertenecer a las producciones artísticas, científicas, sociales o tecnológicas (Ortega et al. 2019).

Para ejercitar la mente creativa hay que comenzar por desarrollar la imaginación, tanto en los aspectos formales como informales. La imaginación y la fantasía tienen una gran importancia dentro de las aulas de clases en todos los niveles, de ahí surgen los proyectos que mejoran al mundo; puede parecer solamente tarea de niños, pero no es así, la imaginación es para adolescentes y adultos, la diferencia radica en que un niño no es propenso a pensar en lo que opinan los demás, mientras que un adulto prefiere verse maduro o expresar ser experto (Hussey, 2017).

Se ha comprobado que la aplicación de la creatividad a nivel cognitivo se presenta desde la consciencia y con tonalidades complejo-evolucionistas, aunque en la práctica queda mucho por hacer, habría que darle la importancia necesaria a la aplicación de la creatividad en las instituciones (Cabrera y De la Herrán, 2015).

Entre los procesos cognitivos relacionados se encuentran pensamientos, imaginación, atención, sensaciones, percepciones, clasificación, asociación, anticipación, abstracción, actividades simbólicas, memoria, lenguaje, codificación e inteligencia (López y Llamas, 2018 y Schrader, 2015). La persona creativa puede describirse como aquella que ha superado la dicotomía entre lo que se considera bueno o malo; las personas autorrealizadas son psicológicamente sanas y maduras, pero no le temen al infantilismo; es común que sean solitarias; generan problemas en una organización, pues están inconformes con diversas situaciones; también son indisciplinados, estrafalarios y poco realistas (Farías, 2017).

La inspiración para desarrollar la creatividad no sólo viene de adentro, sino del espacio en el que el sujeto se desenvuelva y de las oportunidades del espacio que le rodean, a pesar de que la información está al alcance de la mano gracias a las tecnologías de la información y la comunicación, el espacio geográfico es importante para determinada área en la que quiera desempeñarse. De igual manera, un espacio nuevo presenta esas nuevas oportunidades por la novedad que personalmente representa un lugar pequeño o, asimismo, de rutina, puede representar el estancamiento del sujeto creativo (Clapp, 2018). 
La capacidad de asombro que se encuentra entre lo cotidiano y lo sorprendente es mantener la mente abierta, despertar esa sensación de admirar la novedad por aprender, además con ayuda de la imaginación, las actividades dejan de ser rutinarias, se trata de admirar lo que existe alrededor y combinarlo de curiosidad por saber cada vez más sobre algún tema o situación, no importa qué edad se tenga, pues aún no se conoce todo (Egan y Judson, 2018).

La creatividad no es individual, va a depender del contexto en el que se desarrolle, esto es, alguien que concibe una idea y una comunidad que debe aceptar esta idea vinculada a un aspecto nuevo y mejorable, puesto que cualquier ofrecimiento requiere seguidores y además deja de ser invisible cuando se presenta en entornos sociales. En síntesis, el hecho de hacer creatividad significa actuar en el mundo (Clapp, 2018).

En el 2014, Bermejo et al, realizaron un estudio con 240 estudiantes universitarios de entre 18 y 56 años, de la Universidad de Murcia y de la Politécnica de Cartagena, de $1^{\circ}, 2^{\circ}$ y $4^{\circ}$ del grado de Educación Infantil y grado de Educación Primara, así como Grado de Logopedia y Pedagogía e Ingeniería, para analizar el uso y dominio de los procesos cognitivos y creativos. Los estudiantes manifestaron una falta de generación de ideas, bajo pensamiento metafórico-analógico y una baja utilización del proceso de incubación. Al respecto, es importante resaltar que mientras más avanzado el curso, más bajas son sus puntuaciones. Los autores concluyeron que las instituciones debieran utilizar estrategias que favorezcan la práctica de procesos creativos, pues estos van disminuyendo conforme el avance en los estudios.

En 2015, Gregori y Menéndez plantearon un estudio empírico, descriptivo de población mediante encuesta, con los alumnos de 22 años (mediana) recién ingresados a la facultad de Bellas Artes de la Universidad de Barcelona, en el que buscaban indagar en la opinión de los estudiantes acerca del Aprendizaje Basado en Problemas (ABP) y averiguar si los objetivos de las asignaturas coinciden con los objetivos propios del ABP. Los resultados de la investigación sugirieron que podría funcionar el hecho de que los estudiantes de semestres avanzados influyeran sobre los alumnos de nuevo ingreso y viceversa para incrementar su participación y así intensificar 
sus sentimientos de pertenencia a la Universidad. Concluyen que conforme van a avanzando en cursos, los estudiantes van disminuyendo su capacidad de solucionar problemas, así como la colaboración necesaria para trabajar en grupos. Por último, se propone aumentar la identidad institucional en los estudiantes.

De igual manera, en 2015, Rugeles et al. realizaron un trabajo que identifica algunos factores potenciadores tales como: autodisciplina, autoaprendizaje, análisis crítico y reflexivo, así como el trabajo colaborativo. Distinguieron dos tipos de estudiantes, el nativo y el inmigrante digital. Concluyeron que un estudiante virtual, tanto nativo como inmigrante digital, se distingue por los factores potenciadores antes mencionados y esto les permite tener un proceso educativo humanizante y creativo; sin embargo, su desarrollo no sólo consiste en el aumento de infraestructura tecnológica, sino en el beneficio que se puede obtener de ello en ámbitos tanto sociales como académicos, que contribuyan con la difusión del conocimiento para el desarrollo social y humano.

Chiecher, et al. (2018) efectuaron una investigación bajo tres consideraciones: Primero, tomar en cuenta el periodo de extrañamiento por el que pasan los estudiantes de nuevo ingreso, en el que se tienen que adaptar a una nueva etapa de su vida; segundo, reflexionar acerca de la deserción y permanencia de los alumnos; y tercero, promover el desarrollo de la creatividad para su aplicación en la ingeniería- Se concluyó que es notoria la necesidad de desarrollar la creatividad en las áreas de ingeniería, además se encontraron diferencias significativas a favor de las mujeres en las áreas de Literatura, Actuación, Artesanías y Participación Social del Cuestionario de Acciones Creativas (Chiecher, et al., 2018).

Por otra parte, Vergara et al. (2018) realizaron una propuesta acerca de la creatividad en la enseñanza y el aprendizaje del inglés en el nivel superior, que tiene como objetivo realizar una guía metodológica mediante capacitación, que genere cambios para la enseñanza-aprendizaje del idioma inglés con actividades creativas, para ello se llevó a cabo una investigación mixta en la que, con un enfoque cualitativo, se realizaron descripciones, comprensión e interpretación de los procesos y situaciones planteadas en el 
contexto del aprendizaje de inglés por medio de la creatividad y, por otra parte, se aplicaron encuestas de muestreo con un enfoque cuantitativo. Se concluyó que la creatividad aumentaría la calidad educativa.

Asimismo, Álvarez y De Prada (2018) llevaron a cabo un estudio en el que se analizó la medida en la que existe correspondencia entre la formación académica y las exigencias del mercado laboral, desde el punto de vista competencial, con especial atención a la creatividad. Para ello, se examinaron los reportes de las prácticas realizadas en diferentes empresas, puesto que componen el puente entre los estudios universitarios y el mercado laboral. Los resultados obtenidos indican que, tanto alumnos como tutores, en las empresas, tienen un nivel de creatividad con puntuación baja. Las autoras concluyen que la realización de prácticas externas es una excelente oportunidad para que los alumnos conozcan la vida laboral, al tiempo que la universidad se actualiza; sin embargo, este proceso de reajuste debe incluir procesos creativos.

Por otra parte, el éxito escolar implica la preparación para la vida y se corresponde con la idea de alcanzar objetivos y no buscar culpables en la educación, tal como lo hacen los estudios del fracaso escolar, responsabilizando al sistema, contenidos, padres de familia, docentes o alumnos. La condicionante es que, si en los espacios académicos se busca obtener resultados satisfactorios en los productos educativos individuales inmediatos, entonces los estudiantes también se estarán preparando para alcanzar el éxito en otros ámbitos como el profesional, familiar y personal (García, 2012).

Los conceptos relacionados con el éxito académico son tanto la perseverancia y la integración, como el rezago, el abandono y la reprobación, por lo que se deben tomar en cuenta en los perfiles de ingreso y en las trayectorias académicas. Asimismo, el éxito escolar se relaciona con el triunfo y encuentra sus beneficios en el mercado escolar y se define por el capital cultural, es decir, a los hábitos de la vida afines a los conocimientos, habilidades, sensibilidad artística y criterios estéticos que promueven la familia y la escuela (Ortega et al. 2015).

De igual manera, la Organización de las Naciones Unidas para la Educación, la Ciencia y la Cultura (UNESCO) busca que el estudiante 
domine sus áreas de estudio, que es una cualidad que se adquiere a partir del conocimiento y el pensamiento (2015). Las competencias generan un comportamiento exitoso, toman en cuenta los valores y se dividen en cardinales, que son para todos los miembros de la comunidad a la que pertenezca; específicas gerenciales, para los supervisores y específicas por área. Se llevan a cabo gracias al liderazgo, la comunicación eficaz y el trabajo en equipo (Alles, 2015).

El estrés genera desesperanza e incapacidad y daña la salud mental (De la Fuente, 2015), incluso puede estar influenciado por ansiedad generalizada o social, relacionada con algunos eventos que suceden en el ambiente educativo y que posteriormente se refleja en fobia a la escuela, llegando al ausentismo o la deserción por rechazo, lo que trae como consecuencia conducta antisocial y otros problemas familiares y sociales (Cline et al, 2017).

El estudio de Padilla et al. (2017) trata el tema del éxito académico a través de una investigación que abarca la perspectiva de orientadores educativos acerca de cómo los estudiantes de licenciatura viven el primer año de universidad, sus dificultades, y las estrategias que utilizan para solventarlas. Se encontró que los estudiantes experimentan angustia, relacionada con la elección de carrera, y que el tránsito del bachillerato al primer año de estudios universitarios representa un momento en el que definen quiénes son y qué quieren para su vida. Concluyeron que la inducción a la educación universitaria es clave para la integración estudiantil y que las instituciones deben mejorar sus procesos, reconociendo que los estudiantes son agentes activos y atendiendo a áreas socioemocionales. Recomiendan que las instituciones evalúen sus programas de inducción y tutorías para afrontar el reto académico y conocer en qué medida logran los objetivos de disminuir la deserción y contribuir a la adaptación integral de los estudiantes.

De igual manera, Dejo (2015), pretendía realizar una valoración de 156 estudiantes matriculados de la Universidad de Zaragoza. La intención del trabajo de investigación fue analizar la percepción que tuvieran respecto a las competencias que estaban adquiriendo los estudiantes mediante tres actividades, dos con carácter cooperativo y una con carácter individual. Los resultados de los 114 instru- 
mentos válidos mostraron una alta valoración de las competencias adquiridas mediante el aprendizaje cooperativo, que los lleva al éxito académico. Por lo que se concluyó que cada método de estudio requiere de sus propias estrategias para alcanzar las competencias que le corresponden y así asegurar el éxito de los estudiantes, no sólo mientras sean alumnos, sino durante su vida profesional.

Del mismo modo, para establecer una definición integradora de calidad de vida, se toman en cuenta las características principales que le componen, esto es, el bienestar generalizado que comprende el material, físico, psicológico y social, relacionado con el estilo de vida como una propiedad individual, puesto que incluye muestras de seguridad en sí mismo, salud física y productividad personal, entendiéndose en los ámbitos laborales o académicos (Ardila, 2003).

De acuerdo con el glosario de términos del Instituto Nacional de Estadística y Geografía (INEGI, 2018), la calidad de vida se define como un conjunto de elementos que proporcionan tranquilidad y satisfacción a los individuos y a la sociedad en general. Por su cuenta, la Organización para la Cooperación y el Desarrollo Económicos (OCDE), propone 11 temas para definir el bienestar en los ciudadanos: vivienda, ingresos, empleo, comunidad, educación, medio ambiente, compromiso cívico, salud, satisfacción con la vida, seguridad y balance vida-trabajo. Para los mexicanos los tres más importantes son educación, salud y satisfacción con la vida, temas que coinciden con otras regiones y países, sin encontrarse diferencias significativas entre hombres y mujeres (2017).

La manera en la que la sociedad está organizada es por medio de redes, en la actualidad se cuenta con una herramienta poderosa, internet, lo que les da a estas redes un dinamismo flexible para continuar con las relaciones sociales, no sólo con las personas que le rodean sino a nivel global, trascendiendo fronteras, con la capacidad de reproducirse indefinidamente (Puga et al. 2015). La tecnología se ha convertido en un elemento básico para la vida actual y es la principal herramienta para la evolución del ser humano. Existe una brecha tecnológica entre las personas, puesto que quienes tienen acceso a ella promueven su calidad de vida al pertenecer a un sistema productivo, por consiguiente, obtienen un beneficio social; en 
cambio, quienes no pueden acceder a ella se aíslan de la vida actual globalizada (Ortega et al. 2015).

Lo cierto es que sin salud física no es posible realizar las actividades cotidianas, por ello se entiende indispensable para la calidad de vida, así pues, la Organización Mundial de la Salud (OMS) se ha encargado de equiparse con expertos alrededor del mundo para detectar enfermedades y cubrirlas con los medicamentos necesarios. Su objetivo actual es promover la salud en todas las edades, así como el bienestar (2018).

Asimismo, la salud mental se define como un estado de equilibrio, tranquilidad, serenidad, alegría, gusto por vivir. No estaría completa la salud de la persona si no se encuentra con confianza interna, autoestima, manteniendo relaciones significativas y constructivas, satisfacción personal, el estado de ánimo positivo, gozar del silencio, así como éxito académico. La salud mental depende de la adaptación al ambiente, ya que se mantiene estable conforme se desarrollan las potencialidades de la vida, no sólo con la estabilidad emocional (De la Fuente, 2015). La OCDE (2017) arguye que, por la encuesta nacional de calidad de vida, México no tiene estándares de educación altos; sin embargo, las personas están satisfechas con la vida.

La OMS también tiene en cuenta en sus estándares de salud aquellos no transmisibles, como la salud mental, sobre todo cuando se trata de atención en crisis y por violencia, contando el maltrato o la atención a las personas con discapacidades (2018). Es indispensable para la vida cotidiana tener una autoestima sana, ya que dirige el funcionamiento que puede tener el ser humano, desde la apariencia física, no precisamente superficial, ya que es forzoso gustarse a sí mismo; también en el plano psicológico con los procesos mentales que conlleva, tales como, la memoria o la inteligencia y el ambiente sociocultural para las relaciones interpersonales; por eso habría que concentrarse entonces en la autonomía personal, la generosidad o el coraje.

Asimismo, durante 2015, Barrantes y Ureña, aplicaron dos escalas para un estudio no experimental, descriptivo, correlacional y transversal a 402 estudiantes voluntarios de la Universidad Nacional de Costa Rica, con el objetivo de contribuir al conocimiento que 
se tiene sobre el bienestar psicológico y subjetivo de estudiantes universitarios. Del análisis estadístico resultó que los estudiantes tienen niveles altos de bienestar psicológico, sobre todo en las áreas de crecimiento personal, propósito en la vida, satisfacción con la vida por autoaceptación y afecto positivo, mientras que los más bajos están en autonomía y dominio del entorno. El reto que tiene ahora la Universidad es mantener esos estándares y expectativas de los estudiantes.

De esta manera, en el 2015, Batista et al. realizaron un proyecto de promoción de la salud para conocer de qué manera 233 estudiantes que participaron durante cuatro cursos en la Facultad de Biología y de Geografía de la Universidad de la Habana, cuidan su calidad de vida mientras realizan sus estudios en ciencias de la salud. El 70\% de los estudiantes calificó como positivo el curso de las asignaturas electivas para el bienestar personal, esto, a su vez, contribuye con el crecimiento personal y el desarrollo de una comunidad universitaria saludable, por la ética de los estudiantes y el contexto comunitario y social. Se concluyó que ante las actividades que promueven la salud pública se forman hábitos de compromiso social, consigo mismos y con la comunidad.

Igualmente, Franco y Duque (2015) buscaron identificar los significados que tenían para los docentes de la Universidad de Caldas, Manizales, Colombia, el autocuidado en la formación integral de los estudiantes de educación superior como deber bioético. Así que realizaron una investigación de corte cualitativo, formando grupos focales con docentes y por medio de entrevistas no estructuradas, con la finalidad de entender el autocuidado como elemento central de la formación integral y responsabilidad bioética de los profesionales. Para hablar de una responsabilidad bioética la Universidad debe poner en práctica el autocuidado como el elemento más importante de la formación integral para el ámbito bioético.

Por otra parte, se encuentra una investigación sobre estrés académico de Pozos et al. (2015) con el objetivo de identificar un modelo predictivo relacionado con las condiciones de estrés académico que presentan síntomas físicos, psicológicos y comportamentales de 527 estudiantes. Los resultados mostraron que una sobrecarga de 
trabajo académico y participación en clases predijeron síntomas físicos; mientras que la falta de tiempo, unida a los dos primeros, reflejaron síntomas psicológicos y, por último, los trabajos obligatorios, sobrecarga académica, participación en clases y masificación en el aula mostraron síntomas comportamentales.

Igualmente, en 2015, Suárez y Díaz hicieron una revisión sistemática en el Ministerio de Educación Nacional de Colombia y en 18 Universidades en las que la deserción universitaria y las estrategias de retención se han utilizado para contrarrestar el problema a nivel nacional, con la finalidad de identificar la relación entre el estrés académico y la salud mental. Los autores reconocen que no puede haber estrategias de manera integral que terminen con la deserción universitaria a causa del estrés académico, puesto que las necesidades de los estudiantes son muy particulares. El estrés ocasiona depresión, por lo que en su versión académica es causa de la baja salud mental de los estudiantes universitarios, una de las estrategias más utilizadas es el acompañamiento, tanto emocional como psicopedagógico, para atender situaciones individuales.

Del mismo modo, Lara et al. (2015) hicieron un estudio con el objetivo de identificar la percepción de los estudiantes sobre su salud y calidad de vida relacionada con la satisfacción en el medio universitario. Para la realización del estudio se aplicó un cuestionario de datos sociodemográficos de valoración de los servicios universitarios, satisfacción con los estudios, estrés académico, problemas de salud y calidad de vida auto percibida; de lo que resultó que el $84.1 \%$ se encuentra satisfecho con los estudios, el 70\% valoró positivamente las instalaciones y servicios comunitarios, el 63.9\% tuvo estrés en las dos semanas anteriores al estudio, lo que afectó su salud. La calidad de vida fue mayor para quienes reportaron un buen trato de compañeros y docentes. Los investigadores concluyeron que los aspectos favorables repercuten positivamente en la percepción de la calidad de vida de los estudiantes. 


\section{Metodología}

En La presente investigación tiene una pertinencia oportuna en el contexto estudiantil, ya que en diversas ocasiones se minimizan los problemas y situaciones cotidianas de los alumnos de licenciatura. Pareciera que se trata de una etapa en la vida en la que la mayor prioridad son los estudios, olvidándose de los hábitos de salud, tanto física como bienestar psicológico, es decir, divertirse, tener amigos o pareja fuera una rutina insana y las distracciones arruinaran su vida.

Podría creerse que el estudiante debe olvidarse de aquello que le distraiga de sólo dedicarse a estudiar y desarrollar proyectos académicos para tener éxito académico, dejando de lado la calidad de vida, por ello, la importancia de hablar de creatividad e incluir sus indicadores, para que de esa manera exista un equilibrio en la calidad de vida de los estudiantes universitarios, y así alcanzar el éxito académico que será la preparación para que posteriormente alcancen triunfos en la vida profesional. Siguiendo esta problemática, se plantea la siguiente interrogante: ¿Cuál es la situación actual del estudiante universitario respecto al empleo de la creatividad para lograr simultáneamente el éxito académico y la calidad de vida?, de la que se desprende el objetivo de analizar descriptivamente, la creatividad, éxito académico y calidad de vida, para identificar la situación actual del estudiante universitario.

\section{Tipo de investigación}

El trabajo de investigación fue no experimental, de tipo transeccional y descriptivo, argumentando que se midieron tres ejes de investigación: creatividad, éxito académico y calidad de vida, en el contexto en el que suceden, sin modificación alguna, para conocer su incidencia en este ambiente en particular como herramienta de estudiantes universitarios, para así ofrecer una descripción de lo que sucede en la rutina (Hernández et al. 2010), mediante el instrumento de investigación con escala de 1 a 10, lo que viven y 
realizan en su vida cotidiana, no se va a interponer modificación de conducta alguna.

Las variables que se generaron para el trabajo de investigación se presentan con la finalidad de obtener una descripción de la población participante, se consideraron las características de los sujetos correspondientes a los datos generales, mediante variables nominales y ordinales tales como: sexo, edad, estado civil, semestre, lugar de procedencia, programa académico y promedio del semestre anterior.

Se utilizó un instrumento de investigación original que se ha titulado CEECaVi, siendo las iniciales de cada uno de los tres ejes de investigación: creatividad, éxito académico y calidad de vida. Este instrumento se diseñó mediante la información recopilada de artículos científicos arbitrados que se publicaron entre 2015 y 2018. Para establecer los atributos en variables simples y con ello las definiciones con las que se operacionalizaron las variables, se crearon cuatro secciones: una para datos personales, a los que se les dio un carácter politómico, y las otras tres secciones para los ejes de investigación, que se medirán mediante una escala de 1 a 10, de acuerdo con la frecuencia con la que alumnos una Universidad pública de México realizan ciertas actividades encaminadas a su rutina, tanto como estudiantes o en cualquier rol que jueguen socialmente.

El cuestionario fue validado por jueces expertos y pilotado por una población similar a la muestra a la que se aplicó; cabe señalar que, al momento de la aplicación, se ha notificado a los participantes que este instrumento tiene fines investigativos que no afectan su desempeño dentro de la Universidad y es confidencial, por lo que se les pidió que respondieran de manera objetiva y sincera. La población seleccionada para la investigación fueron estudiantes de tres programas de licenciatura de una Universidad pública de México, mediante selección sistemática de elementos muestrales. El total de alumnos es de 248 y de ahí se extrajo la muestra conformada por 151 estudiantes de acuerdo con el algoritmo de Isaac y William (1996) y Krejcie y Morgan (1970). 


\section{Resultados}

Quero (2010) menciona que la fiabilidad de un instrumento debe ser mayor a 0.8. En el instrumento CEECaVi, se obtuvo como resultado un Alfa de Cronbach de 0.887 para un total de 181 elementos diversificados en datos generales y ejes de investigación, lo que significa que es confiable y puede aplicarse a la muestra de la población elegida (ver tabla 1).

Tabla 1. Fiabilidad de CEECaVi

\begin{tabular}{ccc}
\hline Eje & Alfa de Cronbach & $\mathbf{N}^{\circ}$ de elementos \\
\hline Creatividad & .927 & 41 \\
Éxito Académico & .808 & 71 \\
Calidad de Vida & .836 & 54 \\
\hline Total & .887 & 181 \\
\hline
\end{tabular}

Nota: $\mathrm{n}=17$, fuente: CEECaVi

La muestra estudiada estuvo integrada por un $62.2 \%$ (102) de mujeres y un $37.8 \%$ (62) de varones; de los que, 36\% (59) sujetos de investigación, pertenecen al programa de psicología, 32.3\% (53) son parte de la licenciatura en enfermería y $31.7 \%$ (52) de las ingenierías. Se caracterizaron de la siguiente manera: del 100\% (164) de estudiantes universitarios, el $31 \%$ (51) pertenecen al $4^{\circ}$ semestre; el $26.2 \%$ (43) a $8^{\circ}$; el $21.3 \%$ (35) a $2^{\circ}$; el $12.2 \%$ (20) de $6^{\circ}$ semestre; $7.9 \%$ (13) de $10^{\circ}$ semestre. Respecto al lugar de procedencia, el 43.3\% (71) pertenecen a Jalpa, municipio en el que se encuentra el campus en el que estudian, mientras que $56.7 \%$ (93) son foráneos; con lo que respecta al estado civil, el $82.3 \%$ (135) son solteros, el 4.3\% (7) están casados y el 13.4\% (22) mantienen una relación estable sin matrimonio (ver tabla 2). 
Tabla 2. Estadísticos descriptivos de la frecuencia de estudiantes universitarios según semestre

\begin{tabular}{|c|c|c|}
\hline Variables & Frecuencia & Porcentaje (\%) \\
\hline Sexo: & & \\
\hline Mujeres & 102 & 62.2 \\
\hline Hombres & 62 & 37.8 \\
\hline \multicolumn{3}{|l|}{ Programa académico } \\
\hline Psicología & 59 & 36.0 \\
\hline Enfermería & 53 & 32.3 \\
\hline Ingenierías & 52 & 31.7 \\
\hline \multicolumn{3}{|l|}{ Semestre } \\
\hline 2 & 35 & 21.3 \\
\hline 4 & 51 & 31.1 \\
\hline 6 & 20 & 12.2 \\
\hline 8 & 43 & 26.2 \\
\hline 10 & 13 & 7.9 \\
\hline \multicolumn{3}{|l|}{ Lugar de procedencia } \\
\hline Municipio Jalpa & 71 & 43.3 \\
\hline Municipios Colindantes & 93 & 56.7 \\
\hline \multicolumn{3}{|l|}{ Estado Civil } \\
\hline Soltero & 135 & 82.3 \\
\hline Casado & 7 & 4.3 \\
\hline En una relación & 22 & 13.4 \\
\hline Total & 164 & 100.0 \\
\hline
\end{tabular}

Nota: $\mathrm{n}=164$, fuente: $\mathrm{CEECaVi}$

De los resultados de un análisis de estadísticos descriptivos del eje creatividad, se obtiene que la significancia de las variables es menor a 0.050, asimismo, los puntajes de la media permiten afirmar que los estudiantes se distinguen en utilizar indicadores de la creatividad, ya que tienen curiosidad, están dispuestos a probar nuevas formas de aprendizaje, han modificado su conducta así como su punto de vista, superan sus calificaciones cada semestre, modifican sus ideas de acuerdo a las situaciones, realizan sus tareas, tienen habilidades 
por desarrollar, se establecen metas académicas, son competentes, analizan diferentes propuestas para elegir una correcta y toman en cuenta su experiencia previa para inventar algo nuevo (ver tabla 3).

Tabla 3. Análisis descriptivo del eje Creatividad

\begin{tabular}{|c|c|c|}
\hline Variables & $\bar{X}$ & $\mathbf{P}$ \\
\hline 30. Tengo curiosidad por conocer cosas nuevas & 9.4 & 0 \\
\hline 26. Estoy dispuesto a probar nuevas formas de aprendizaje & 8.95 & 0 \\
\hline $\begin{array}{l}\text { 27. He modificado mi conducta conforme cambio de nivel } \\
\text { educativo }\end{array}$ & 8.9 & 0 \\
\hline 12. Me propongo superar mis calificaciones cada semestre & 8.84 & 0 \\
\hline $\begin{array}{l}\text { 4. Tengo la disposición para modificar mis ideas de acuerdo } \\
\text { a las situaciones }\end{array}$ & 8.74 & 0 \\
\hline 3. Me comprometo a realizar tareas & 8.71 & 0 \\
\hline 24. Considero que tengo habilidades por desarrollar & 8.57 & 0 \\
\hline 11. Me establezco metas académicas cada semestre & 8.45 & 0 \\
\hline 29. Soy competente en el área de mis habilidades & 8.41 & 0 \\
\hline $\begin{array}{l}\text { 18. Analizo mis diferentes propuestas para elegir una } \\
\text { correcta }\end{array}$ & 8.29 & 0 \\
\hline 10. Modifico mi punto de vista académico & 8.05 & 0 \\
\hline $\begin{array}{l}\text { 21. Tomo en cuenta mi experiencia previa para inventar } \\
\text { algo nuevo }\end{array}$ & 8.05 & 0 \\
\hline
\end{tabular}

Nota: $\bar{X}=$ media, $\mathrm{P}=$ Significancia asintótica (bilateral), $\mathrm{n}=164$, ${ }^{*} \mathrm{p}<0.050$, fuente: CEECaVi

Asimismo, los resultados del análisis de estadísticos descriptivos del eje éxito académico tienen una significancia menor a 0.050 , también se demuestra que los puntajes de la media ratifican que los estudiantes se distinguen por ser responsables de sus éxitos así como de sus fracasos, persisten en estudiar su carrera, les gusta buscar nuevas experiencias, se perciben capaces de lograr lo que se proponen cuando se trata de cuestiones académicas y procuran profundizar en los contenidos que les resultan interesantes. 
De igual manera, enfrentan tareas que generan estrés, disfrutan aprender por su cuenta, disfrutan del presente más que del pasado o del futuro, el trabajo a conciencia les resulta satisfactorio. Los estudiantes son capaces de superar sus expectativas cada semestre, cuentan con las habilidades que se requieren para realizar las prácticas necesarias, sus logros se equiparan con lo que se propusieron al iniciar el ciclo académico, cooperan en actividades colaborativas con otros alumnos y evalúan de manera personal sus éxitos académicos (ver tabla 4).

Tabla 4. Análisis descriptivo del eje Éxito Académico

\begin{tabular}{|c|c|c|}
\hline Variables & $\bar{x}$ & $\mathbf{P}$ \\
\hline 47. Soy responsable de mis éxitos, así como de mis fracasos & 9.29 & 0 \\
\hline 39. Persisto en estudiar esta carrera & 9.19 & 0 \\
\hline 61. Me gusta buscar nuevas experiencias & 8.73 & 0 \\
\hline $\begin{array}{l}\text { 49. Me percibo capaz de lograr lo que me propongo cuando } \\
\text { se trata de cuestiones escolares }\end{array}$ & 8.68 & 0 \\
\hline $\begin{array}{l}\text { 66. Procuro profundizar en los contenidos que me resultan } \\
\text { interesantes }\end{array}$ & 8.49 & 0 \\
\hline 41. Enfrento tareas que generan estrés & 8.46 & 0 \\
\hline 65. Disfruto aprender por mi cuenta & 8.41 & 0 \\
\hline 60. Disfruto del presente más que del pasado o del futuro & 8.38 & 0 \\
\hline 45. Soy capaz de superar mis expectativas cada semestre & 8.32 & 0 \\
\hline 64. El trabajo a conciencia me resulta satisfactorio & 8.32 & 0 \\
\hline $\begin{array}{l}\text { 37. Cuento con las habilidades que se requieren para realizar } \\
\text { las prácticas necesarias }\end{array}$ & 8.27 & 0 \\
\hline $\begin{array}{l}\text { 48. Mis logros se equiparan con lo que me propuse al iniciar } \\
\text { el ciclo escolar }\end{array}$ & 8.15 & 0 \\
\hline 38. Coopero en actividades colaborativas con otros alumnos & 8.12 & 0 \\
\hline 50. Evalúo de manera personal mis éxitos escolares & 8.11 & 0 \\
\hline
\end{tabular}

Nota: $\bar{X}=$ media, $\mathrm{P}=$ Significancia asintótica (bilateral), $\mathrm{n}=164$, ${ }^{*} \mathrm{p}<0.050$, fuente: CEECaVi 
Y, por último, los resultados del análisis de estadísticos descriptivos del eje calidad de vida arrojan que todas las variables son mayores a 0.050 , de la misma forma los puntajes de la media afirman que los estudiantes se distinguen por sentir la necesidad de salir adelante por cuenta propia, responder por sus acciones, utilizar internet y celular para la vida cotidiana (incluyendo cuestiones académicas y tiempo de ocio), hacer uso de las TIC para preparar una exposición, les gusta ser estudiantes, valoran su trabajo, la computadora es su herramienta de trabajo, reconocen sus habilidades y limitaciones, se sienten felices, se gustan a sí mismos y ven el lado positivo de las situaciones (ver tabla 5).

Tabla 5. Análisis descriptivo del eje Calidad de vida

\begin{tabular}{lcc}
\hline \multicolumn{1}{c}{ Variables } & $\bar{X}$ & $\mathbf{P}$ \\
\hline $\begin{array}{l}\text { 79. Siento la necesidad de salir adelante por cuenta mía } \\
\text { 69. Utilizo el internet para la vida cotidiana (incluyendo }\end{array}$ & 9.27 & 0 \\
$\quad$ cuestiones escolares y tiempo de ocio) & 9.26 & 0 \\
77. Respondo por mis acciones & 9.26 & 0 \\
71. Hago uso de las TIC para preparar una exposición. & 9.25 & 0 \\
70. Utilizo el celular para la vida cotidiana (incluyendo & 9.15 & 0 \\
cuestiones escolares y tiempo de ocio) & & \\
74. Me gusta ser estudiante & 9.11 & 0 \\
82. Valoro mi trabajo & 9.05 & 0 \\
72. La computadora es mi herramienta de trabajo & 8.88 & 0 \\
81. Reconozco mis habilidades y limitaciones & 8.84 & 0 \\
73. Me siento feliz & 8.51 & 0 \\
78. Me gusto a mí mismo & 8.44 & 0 \\
76. Veo el lado positivo de las situaciones & 8.34 & 0 \\
\hline
\end{tabular}

Nota: $\bar{X}=$ media, $\mathrm{P}=$ Significancia asintótica (bilateral), $\mathrm{n}=164$, ${ }^{*} \mathrm{p}<0.050$, fuente: CEECaVi 


\section{Conclusiones}

Se responde a la necesidad de llevar a cabo actividades basadas en los indicadores de la creatividad en diferentes ámbitos, tanto académico como de la vida cotidiana, así como se muestra en los resultados. La situación actual del estudiante universitario, respecto al empleo de la creatividad para lograr simultáneamente el éxito académico y la calidad de vida, se refleja que el uso que le dan a la primera, con referencia a sus indicadores, de los que el más aceptado es la curiosidad y la flexibilidad para modificar conductas y perspectivas con miras a superar retos autoimpuestos, abiertos a conocer puntos de vista diferentes, encuentran la motivación en realizar proyectos con la necesidad de continuarla así como diseñar nuevas estrategias, e inconformismo con la vida cotidiana, la búsqueda de nuevas metas y la apertura al aprendizaje.

De igual manera, los estudiantes reflejan que para alcanzar el éxito académico se requiere de la responsabilidad, es decir, de un locus de control interno, puesto que las situaciones que viven son consecuencias de sus actos. Los estudiantes están conscientes de sus capacidades y el trabajo en equipo, además expresan la dificultad que les representa estudiar y el estrés académico al que están sometidos, pero lo contrarrestan perseverando y con autoaprendizaje. Asimismo, se nota la tendencia a la innovación, la búsqueda del éxito en sus proyectos, ya que se perciben como estudiantes con las habilidades necesarias para profundizar en los contenidos que más les interesan, sin necesidad de que el profesor lo asigne como tarea obligatoria.

Es notoria la importancia del uso de diferentes software y hardware para la vida cotidiana de los estudiantes universitarios, usos que van desde las tareas académicas, hasta el tiempo de uso de estos recursos en actividades de ocio, observándose una necesidad y gusto por el uso de la tecnología para cualquier ámbito de su vida, ya que se trata de nativos digitales.

Por otra parte, se distingue la perseverancia en los alumnos, se puede describir a jóvenes con autoestima y optimismo que se 
esfuerzan por conseguir lo que quieren y se hacen responsables de las consecuencias de sus decisiones, así como de sus actos. Hay una constante búsqueda de autorrealización por medio de valores como la responsabilidad, de igual manera se percibe un autorreconocimiento y satisfacción con la vida universitaria.

Sobre la base de estos resultados y de acuerdo con la evidencia empírica recabada, se plantea que la creatividad de los estudiantes de educación superior promueve el logro del éxito académico y las herramientas para alcanzar la calidad de vida. Se concluye también que a los alumnos les hacen falta espacios de tiempo libre con referencia al descanso y el esparcimiento para promover las interrelaciones, tanto amistosas como de pareja, así como mayores herramientas tecnológicas desde la institución y la promoción de la salud mental, no sólo cuando exista un problema mayor sino con carácter de prevención para un bienestar psicológico.

\section{Referencias}

Alles, M. A. (2015). Diccionario de Competencias: las 60 competencias más utilizadas en gestión por competencias. Tomo 1. Buenos Aires: Granica.

Álvarez Santullano, M. M. y De Prada Creo, E. (2018). Evaluación de las competencias profesionales a través de las prácticas externas: incidencia de la creatividad. Revista de Investigación Educativa, 36, 203-219.

Ardila, R. (2003). Calidad de Vida: Una definición Integradora. Revista Latinoamericana de Psicología, 2, 161-164.

Barrantes Brais, K., y Ureña Bonilla, P. (2015). Bienestar psicológico y bienestar subjetivo en estudiantes universitarios costarricenses. Revista Intercontinental de Psicología y Educación, 17, 101-123.

Batista Mainegra, A., Rojas Hernández, N. M., González Aportela, O., y Hernández García, L. (2015). Educación para la Salud desde el currículum electivo como experiencia de formación integral de estudiantes universitarios. Revista Cubana De Educación Médica Superior, 29, 555-565.

Bermejo García, R., Ferrando Prieto, M. M., Sainz Gómez, M., Soto Martínez, G., y Ruiz Melero, M. J. (2014). Procesos Cognitivos de la Creatividad 
en Estudiantes Universitarios. Educatio Siglo XXI, 32, 41-58.

Cabrera Cuevas, J. D. y De la Herrán Garcón, A. (2015). Creatividad, complejidad y formación: un enfoque transdisciplinar. Revista complutense de educación, 26, 505-526.

Chiecher, A. C., Elisondo, R. C., Paolini, P. V. y Donolo, D. S. (2018). Creatividad, género y rendimiento académico en ingresantes de ingeniería. Revista Iberoamericana de Educación Superior, 24, 138-151.

Clapp, E. P. (2018). La Creatividad como proceso participativo y distribuido. Madrid: Narcea.

Cline, T., Gulliford, A. y Birch, S. (2017). Psicología Educativa. Ciudad de México: Editorial Manual Moderno.

De la Fuente, R. (2015). Psicología Médica. Ciudad de México: Fondo de Cultura Económica.

Dejo Oricain, N. (2015). Adquisición de competencias en el marco del Aprendizaje Cooperativo: valoración de los estudiantes. Revista de Docencia Universitaria, 1, 339-359.

Egan, K. y Judson, G. (2018). Educación Imaginativa. Madrid: Narcea.

Farías Carracedo, C. (mayo de 2017). Creatividad: Un camino posible para la Educación. En Ortega Neri (Coordinación). $2^{\circ}$ Foro de Creatividad. Conferencia llevada a cabo en Zacatecas, México.

Franco Peláez, Z. R., y Duque Escobar, J. A. (2015). La bioética y el autocuidado de la salud: imperativos para la formación integral en la universidad. Acta Bioéthica, 21, 37-44.

García Martínez, J. (2012). Análisis y claves del éxito escolar: una reflexión compartida. EDETANIA, Vol 42 (1) pp. 189-200.

Gregori Giralt, E., y Menéndez Varela, J. (2015). La percepción de los estudiantes de bellas artes sobre lo aprendido en un entorno de aprendizaje basado en problemas. (Spanish). Revista Mexicana de Investigación Educativa, 20 (65), 481-506.

Hernández Sampieri, R., Fernández Collado, C. y Baptista Lucio, P. (2010). Metodología de la Investigación. Ciudad de México: McGraw Hill.

Hussey, W. (2017). Pensamiento Creativo. Actividades estimulantes, increibles!, para niños de 6 a 12 años. Madrid: Narcea.

Instituto Nacional de Estadística y Geografía. (2018). Datos Glosario. México: INEGI. Recuperado de: http://www.beta.inegi.org.mx/app/glosario/ default.html?p=ENSI2010\#letraGloC. 
Isaac, S. y William, B. (1996). Handbook in Research and Evaluation. A collection of principles, methods, and strategies useful in plannieng, design and evaluation studies in educational and behavioral sciences. Second Edition. San Diego: Edits Publishers.

Krejcie, R. V. y Morgan, D. W. (1970). Determining sample size for research activities. Educational and Psychological Measurement, 30, 607-610.

Lara Flores, N., Saldaña Balmori Y., Fernández Vera, N. y Delgadillo Gutiérrez, H. J. (2015) Salud, calidad de vida y entorno universitario en estudiantes mexicanos de una universidad pública. Hacia promoc. Salud, 20(2): 102-117.

López Fernández, V. y Llamas Salguero, F. (2018). Neuropsicología del proceso creativo. Un enfoque Educativo. Revista Complutense de Educación, 29(1), 113-127.

Organización de las Naciones Unidas para la Educación, la Ciencia y la Cultura (2015). Marco Conceptual para la evaluación de las competencias. Ciudad de México: UNESCO. Recuperado de: http://www.ibe.unesco.org/ sites/default/files/resources/ipr4-roegiers-competenciesassessment_ spa.pdf.

Organización Mundial de la Salud. (2018). Folleto. París: OMS. Recuperado de: http://www.who.int/es/about/what-we-do/folleto.

Organización para la Cooperación y el Desarrollo Económicos. (2017). ¿Cómo va la vida en México? México: OCDE. Recuperado de https://www. oecd.org/statistics/Better-Life-Initiative-country-note-Mexico-in-Espagnol.pdf.

Ortega Guerrero, J. C., López González, R. y Alarcón Montiel, E. (2015). Trayectorias escolares en educación superior. Propuesta metodológica y experiencias en México. Xalapa: Universidad Veracruzana.

Ortega Neri, H. M., Pérez Márquez, E. A. y Acosta De Lira, J. A. (2019). Psicología de la creatividad aplicada. Ciudad de México: Colofón.

Padilla González, L. E., Figueroa Ruvalcaba, A. E., y Rodríguez-Figueroa, H. M. (2017). La incorporación a la universidad de los estudiantes en Aguascalientes. La perspectiva del orientador educativo. Sinéctica, Revista electrónica de educación, 48, 77-97.

Pozos Radillo, B. E., Preciado Serrano, M. L., Plascencia Campos, A. R., Acosta Fernández, M. y Aguilera V., M. A. (2015). Estrés Académico y Síntomas Físicos, Psicológicos y Comportamentales en estudiantes mexicanos de 
una Universidad Pública. Ansiedad y Estrés 21, 35-42.

Puga, C., Peschard, J. y Castro, T. (2015). Hacia la sociología. Ciudad de México: Pearson.

Quero Virla, M. (2010). Confiabilidad de coeficiente Alfa de Cronbach. Revista Estudios Interdisciplinarios en Ciencias Sociales, 12, 248-252.

Rugeles Contreras, P. A., González, B. M., y Metaute Paniagua, P. M. (2015). El rol del estudiante en los ambientes educativos mediados por las TIC. Revista Lasallista De Investigación, 12, 132-138.

Schrader, C. [TEDxUniSabana] (2015, marzo 17). Los Procesos de Pensamientos Creativos en la Educación. Recuperado de: https://www.youtube.com/watch?v=FhO_gs_3drg\&list=PL4aXuHQrLoCLBhcYiC9-_ccmENS3kyMcA\&index=3.

Suárez Montes, N., y Díaz Subieta, L. B. (2015). Estrés académico, deserción y estrategias de retención de estudiantes en la educación superior. Revista De Salud Pública, 17, 300-313. doi:10.15446/rsap. v17n2.52891.

Vergara Mendoza, Z., Navarrete Cornejo, A. y Morán Sánchez, N. (2018). La creatividad en el proceso de enseñanza aprendizaje del idioma inglés a nivel superior. Revista Científica de Investigación Actualización del Mundo de las Ciencias, 2, 174-193.

Recibido: 07.07.2020 Aceptado: 16.10.2020 